\title{
Diacronie
}

Studi di Storia Contemporanea

$N^{\circ} 17,1 \mid 2014$

Periferie. Cultura, economia, politica

\section{Légitimer le pouvoir royal suédois à travers une nouvelle construction de l'histoire nationale de Suède}

\section{Muriel Marchal}

\section{(2) OpenEdition \\ Journals}

Édition électronique

URL : http://journals.openedition.org/diacronie/983

DOI : 10.4000/diacronie.983

ISSN : 2038-0925

Éditeur

Association culturelle Diacronie

Référence électronique

Muriel Marchal, « Légitimer le pouvoir royal suédois à travers une nouvelle construction de l'histoire nationale de Suède », Diacronie [En ligne], № 17, 1 | 2014, document 1, mis en ligne le 01 mars 2014, consulté le 10 décembre 2020. URL : http://journals.openedition.org/diacronie/983 ; DOI : https:// doi.org/10.4000/diacronie.983 


\title{
Diacronie
}

\section{Légitimer le pouvoir royal suédois à travers une nouvelle construction de l'histoire nationale de Suède}

\author{
Muriel MARCHAL *
}

Charles XI de Suède accéda au trône de Suède après une longue période de régence, qui fut marquée par une période de paix avec le Danemark. Sa position avait été affaibli par la noblesse, et il avait besoin de consolider sa légitimité royale. Il décida de se servir de l'historiographie nationale pour se créer une bonne image de souverain à travers une nouvelle construction de l'histoire nationale de Suède. Le roi s'appuya notamment sur Atlantica d'Olaus Rudbeck, une nouvelle histoire suédoise.

\begin{abstract}
On cite de temps en temps un ouvrage composé en Suede depuis peu par Olaus Rudbeckius. C'est un livre infolio intitulé Atlantica, qui nous n'avons encore vu. On nous le fait esperer au premier jour. On nous dit qu'il est plein de belles choses, \& ceux qui savent que Mons: Rudbeckuis s'est engage de montrer que presque tous les peuples dont les histoires nous parlent, sont originaires de Suède, ont beaucoup d'impatience de voir comment il prouve ce paradoxe.
\end{abstract}

Article de la République des Lettres, octobre $1684^{1}$

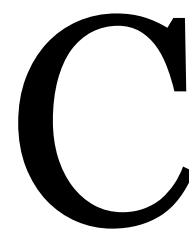

et article de la République des Lettres, publié en octobre 1684, fait mention de la récente publication du premier volume d'Atlantica par Olaus Rudbeck. Spécialiste en anatomie et en botanique, Olaus Rudbeck est l'un des plus grands scientifiques suédois du XVIIème siècle. Il a également été historiographe et a écrit la plus grande œuvre dite historique sur la Suède et sa

1 Extrait de l'introduction de RUDBECK, Olaus, Atlantica, vol.1 [facsimile], Uppsala-Stockholm, Almqvist \& Wiksell, 1937, p. 5 . 
splendeur passée, Atlantica ${ }^{2}$. Dans cet ouvrage, il associe ses connaissances scientifiques novatrices et l'histoire de Suède pour démontrer que le royaume de Suède était le plus ancien et le plus prestigieux.

La Suède a obtenu son indépendance par rapport au Danemark en 1523 et a gagné rapidement de l'importance sur la scène européenne pendant la guerre de Trente Ans en Allemagne, lors des grandes victoires militaires de Gustave II Adolphe. Depuis cette séparation, le royaume de Suède était en état de guerres, en particulier contre le Danemark, pour pouvoir s'affirmer militairement et diplomatiquement. À cause des menaces constantes et des différentes guerres, le souverain suédois avait besoin de posséder une position forte à la tête de leur royaume, pour avoir le soutien de la population dans l'effort de guerres et des grandes cours européennes ${ }^{3}$.

C'est dans ce contexte d'affirmation et de grandeur suédoises, qu'Olaus Rudbeck publia cette œuvre en quatre volumes entre 1679 et 1702 , année de sa mort. Il commença la rédaction d'Atlantica au début du règne de Charles XI, après une longue période de régence et pendant la guerre de Scanie (1676-1679), opposant la Suède au Danemark. Fils de Charles X Gustave (1622-1660), Charles XI eut de nombreuses difficultés à reprendre le pouvoir au Conseil du Royaume, qui s'était renforcé pendant la régence, et, malgré sa noble descendance et son droit héréditaire au trône, à affirmer sa légitimité, pour pouvoir asseoir son pouvoir royal.

\section{La Suède du XVIIème siècle}

La Scandinavie n'était pas à cette époque un ensemble pacifique et unifié. L'Union de Kalmar avait unifié les trois royaumes scandinaves sous l'autorité du souverain danois et était tombée en 1523, après les révoltes suédoises. L'hostilité interne entre le Danemark et la Suède devint une rivalité militaire ouverte sur la scène européenne. Cette longue période sous l'autorité danoise était considérée par les Suédois comme un véritable traumatisme, sur lequel la nouvelle identité suédoise s'est construite. Ce traumatisme eut une grande importance dans les rapports entre le Danemark et la Suède, marquant fortement leurs relations diplomatiques et orientant leurs politiques étrangères respectives ${ }^{4}$.

2 RUDBECK, Olaus, Atlantica, 4. voll., [facsimile] Uppsala-Stockholm, Almqvist \& Wiksell, 1937-1939 [Ed. orig., Atlantica, 4. voll., Uppsala, 1679-1702].

3 BATTAIL, Jean-François, BOYER, Régis, FOURNIER, Vincent, Les sociétés scandinaves: de la Réforme à nos jours, Paris, Presses universitaires de France, 1992.

4 NORDMANN, Claude, Grandeur et liberté de la Suède (166o-1792), Paris-Louvain, Nauwelaerts, 1971. 
Entre 1563 et 1720, eut lieu sept grandes guerres en Scandinavie, qui opposèrent le Danemark et la Suède. La rivalité entre ces deux royaumes dépassa le cadre militaire et diplomatique pour se répandre rapidement dans tous les domaines, en particulier scientifique et culturel. Le Danemark était considéré comme l'ennemi héréditaire de la Suède et le pouvoir en place, au fil des différents règnes, attisait la haine du peuple contre cet ennemi pour pouvoir asseoir durablement la position du souverain suédois. Nous retrouvons ce phénomène plus particulièrement pendant la période de grandeur de la Suède, qui correspond aux règnes des rois Charles X Gustave, Charles XI et Charles XII.

Pendant tout le règne de Charles $\mathrm{X}$ Gustave, le royaume de Suède fut en guerre contre la Pologne, la Russie et le Danemark ${ }^{5}$. Sa mort en 1660 marqua la fin de toutes ces guerres et le début d'une longue période de paix pendant la régence du souverain mineur, le futur Charles XI. En 1675, Charles XI fut couronné roi de Suède. La même année, le roi danois Christian $\mathrm{V}$ déclara à nouveau la guerre à la Suède, notamment dans le but de récupérer les provinces que le Danemark avait perdu lors de la paix de Copenhague en 1660. La guerre de Scanie, qui dura jusqu'en 1679, ne fut ni une victoire pour la Suède, ni une défaite pour le Danemark, mais fut marquée par l'affirmation des limites de la puissance militaire suédoise. Cependant, le souverain suédois en était sorti renforcé, en particulier au niveau de son prestige personnel. Pour cela, Charles XI avait été obligé de mettre en place une propagande directe à l'intention de la population. Il fallait que le roi redonne à son peuple la fierté d'appartenir à ce royaume ${ }^{6}$.

Après sa victoire contre les Danois lors de la bataille de Lund en 1679, Charles XI put profiter d'une grande popularité, auréolée de ce succès militaire. Il profita également du mécontentement de la population contre la noblesse, pour l'affaiblir et mettre en place l'absolutisme en 1680 dans tout le royaume de Suède. Il pouvait s'appuyer sur le prestige de son ascendance royale et sur ses ancêtres, ainsi que sur leurs actes héroïques pour affirmer son pouvoir. Après la longue période de domination danoise, le royaume de Suède avait besoin de se reconstruire une nouvelle image, tant au niveau national qu'auprès des grandes cours européennes. Le but était de retrouver la splendeur passée du royaume de Suède et de faire oublier ce passé commun avec le Danemark, en prouvant sa supériorité. Pour devenir une grande puissance parmi les grandes puissances européennes déjà établies historiquement en Europe, il fallait

\footnotetext{
5 LOCKHART, Paul Douglais, Sweden in the seventeenth century, London, Palgrave, 2004. ${ }^{6}$ SCHNAKENBOURG, Éric, MAILLEFER, Jean-Marie, La Scandinavie à l'époque moderne, fin XVème-début XIXème siècle, Paris, Belin, 2010.
} 
absolument posséder une histoire nationale sur laquelle le souverain suédois pouvait se référer pour prouver la légitimité de la place qu'il demandait à occuper au sein de l'Europe. S'appuyer sur un prestige national important pour le présenter au reste de l'Europe était l'élément principal de cette construction, et l'histoire y jouait un rôle clé dans cette perspective politique.

\section{Le mouvement göticiste en Suède}

Ces guerres entre le Danemark et la Suède dépassèrent rapidement le cadre militaire pour s'étendre aux domaines culturel et scientifique. Sous cette impulsion, apparut dans ces deux royaumes un patriotisme culturel et linguistique très virulent, le mouvement göticiste. Tant au Danemark qu'en Suède, tout fut mis en place pour démontrer que l'histoire, la culture et le passé de son royaume étaient supérieurs à ceux de son voisin, pour pouvoir s'affirmer sur la scène européenne, tant d'un point de vue culturel que diplomatique7.

Le mouvement göticiste consistait à retrouver les racines du pays, ainsi que son passé et le rendre le plus glorieux possible afin d'affirmer sa supériorité par rapport à tous les autres pays concurrents. Une réaction patriotique exalta, particulièrement face à l'invasion étrangère, les vieilles vertus nordiques de bravoure et d'ascétisme. Ce fut là un des thèmes récurrents du göticisme suédois. Il se basait sur la théorie qui voulait faire de la Suède le berceau des peuples et des cultures d'Europe. Ce mouvement consistait à revenir aux racines nationales, en se basant sur le passé de la Suède, comme les Vikings, la mythologie nordique et plus particulièrement les Goths, qui étaient considérés comme l'un des peuples les plus évolués, afin de permettre au passé national d'être le plus glorieux possible. Le but principal était d'affirmer sa supériorité par rapport à son ennemi.

Le mythe göticiste faisait des Suédois les descendants directs des Goths. Dans les premières mentions de ce peuple, ils y sont décrits comme un peuple germanique regroupé en un royaume solide. Ils étaient de fiers et braves guerriers, qui n'avaient peur de rien et qui voyageaient beaucoup, à la découverte de nouveaux territoires. C'est de là que venait le nom du mouvement göticiste, notamment parce qu'à l'étranger, la Suède était appelée Gothia ${ }^{8}$.

\footnotetext{
7 BATTAIL, Jean-François, La Suède intellectuelle et savante, Uppsala, Uppsala Studies in History of Science, 1987.

8 DELBLANC, Sven, LONNROTH, Lars, Den Svenska Litteraturen: från runor till romantism, volume 1, Stockholm, Albert Bonnier, 1999.
} 


\section{Olaus Rudbeck, un scientifique respecté, mais un historien décrié}

Ce mouvement göticiste atteignit son apogée en Suède en la personne d'Olaus Rudbeck. Personnage hors du commun dans toute l'histoire suédoise, Olaus Rudbeck fut professeur de médecine et d'anatomie à l'université d'Uppsala ${ }^{9}$. Il se spécialisa également dans la botanique, ainsi que la géologie, la cartographie, l'architecture, et bien d'autres domaines ${ }^{10}$. Il a laissé de nombreux écrits en suédois, et ses différentes activités scientifiques lui permirent d'acquérir, de son vivant, une grande renommée européenne, devenant ainsi le savant suédois le plus important du XVIIème siècle. Il transforma durablement les pratiques scientifiques, faisant entrer la Suède dans l'ère de la modernité scientifique ${ }^{11}$.

À côté, il développa un intérêt très marqué pour les recherches sur les antiquités et l'histoire suédoises. Il considéra la rédaction d'Atlantica comme l'aboutissement suprême de sa carrière, pouvant mettre ainsi en application les nouvelles méthodes scientifiques, qu'il avait élaboré au début de sa carrière universitaire. Cela lui servit pour démontrer l'exactitude de toutes ses affirmations avec des preuves concrètes et scientifiquement fiables. Le poète romantique suédois Per Daniel Amadeus Atterbom décrivit cette œuvre par ces mots:

si l'Altantica de Rudbeck est une des plus grandes folies que l'histoire ait connues, il faut admettre que cette folie est incomparablement plus intéressante que la sagesse de la plupart des juges. C'est dans cet esprit qu'il faut comprendre Atlantica, tant comme monument national que comme objet de recherches ${ }^{12}$.

Malgré une démarche scientifique et historique rigoureuse, Olaus Rudbeck écrivit cet ouvrage avec des intentions politiques précises. Il estimait que l'histoire était le moyen de diffusion le plus large pour son message politique, à travers ses théories sur l'origine de la Suède et son destin en Europe. La Suède était devenue la grande puissance de l'Europe du Nord en 1660, aux dépens de son voisin danois. Olaus

\footnotetext{
9 BRUSEWITZ, Gunnar, Från Olof Rudbeck till Olof Thunman: 15 Uppsalaprofiler, Uppsala, Uppsala Nya Tidning, 1998.

${ }_{10}$ Rudbecks-studier, Festskrift vid Uppsala Universitets minnesfest till högidlighållande av 30o-årsminnet av Olof Rudbeck d.ä.s födelse, Uppsala, Uppsala Universitet, 1930.

11 DAHL, Per, Svensk ingenjörskonst under stormaktstiden: Olof Rudbecks tekniska undervisning och pratiska verksamhet, Uppsala, Uppsala Universitet, 1995.

${ }_{12}$ ATTERBOM, Per Daniel Amadeus, Minne af professoren i medicinen vid Uppsala universitet Olof Rudbeck den äldre, Stockholm, s. e., 1849, p. 97.
} 
Rudbeck s'était donné pour mission de redécouvrir le passé très prestigieux de la Suède, que l'histoire avait oublié à cause de la domination danoise en Suède. Il voulait redonner à son royaume et à son souverain toutes ses lettres de noblesse, qui devaient lui revenir naturellement, à en croire l'histoire suédoise. Voilà comment nous pouvons expliquer l'apport de l'œuvre Atlantica d'Olaus Rudbeck au nouveau pouvoir suédois de Charles XI.

\section{Charles XI de Suède}

À la mort du roi Charles X Gustave en 1660, son fils Charles XI était âgé de 4 ans et un gouvernement de régence se mit en place jusqu'à la majorité du jeune souverain. Grâce à ce gouvernement, la noblesse suédoise put retrouver sa splendeur passée, qu'elle avait perdue sous le règne de Charles X Gustave, ainsi que le pouvoir absolu sur tout le royaume de Suède. Cette période de régence fut marquée par une paix suédoise avec le reste de l'Europe, et en particulier avec le Danemark. Cependant, elle fut aussi marquée par une propagande très virulente contre les Suédois au Danemark. La Suède était devenue la grande puissance de référence en Europe du Nord, aux dépens du Danemark en 1660, amputant ce royaume par la même occasion de plusieurs de ses régions les plus riches. Le traité de Copenhague, signé en mai 1660, avait développé au sein du Danemark un esprit de revanche très virulent contre les Suédois, dans l'espoir de pouvoir récupérer les provinces du Skåneland, dans le sud de la Suède actuelle, et la place de première puissance d'Europe du Nord ${ }^{13}$.

Couronné le 28 septembre 1675, Charles XI put commencer à gouverner dès 1672, mais le conseil du royaume, composé de la noblesse suédoise, continuait à avoir la main mise sur le pouvoir. Cette omniprésence du conseil empêchait le roi de profiter pleinement des pouvoirs. Charles XI était légalement sur le trône, mais il lui manquait une légitimité pour asseoir son autorité royale au début de son règne. En 1676, le Danemark profita de la mauvaise situation financière de la Suède et du fait que le roi de Suède était déjà en guerre contre la Hollande, aux côtés de son allié français Louis XIV, pour attaquer la Suède le 29 juin 1676. Cela marqua le début de la guerre de Scanie, qui s'acheva en 1679 par la victoire en demi-teinte de la Suède. La Suède avait gagné contre

13 FLORÉN, Anders, Karl X Gustav in FLORÉN, Anders, DAHLGREN, Stellan, LINDEGREN, Jan (red.), Kungar och Krigare, tre essäer om Karl X Gustav, Karl XI och Karl XII, Stockholm, Atlantis, 1992. 
la Hollande, ce qui lui permit d'imposer ses conditions au roi danois, malgré le fait qu'il n'y avait pas eu de véritables vainqueurs dans cette guerre ${ }^{14}$.

Olaus Rudbeck entreprit la rédaction de ce qu'il qualifia l'œuvre de sa vie, Atlantica, à une époque où la Suède se battait pour conserver sa place de grande puissance du Nord sur la scène européenne. Il publia le premier volume en 1679. Cet ouvrage changea l'image de la Suède ${ }^{15}$, donnant au souverain une base historique solide pour légitimer son pouvoir et ses ambitions militaires et diplomatiques pour son royaume.

\section{L'aspect historique d'Atlantica}

Sa démarche première était purement historique ${ }^{16}$. Son ami Olof Verelius, professeur à la chaire des antiquités de l'université d'Uppsala, lui demanda de réaliser en 1672 des cartes de la Suède, afin d'expliquer le cheminement du texte d'une saga islandaise, qu'il voulait publier. Une saga islandaise était un récit originaire d'Islande, qui avait été écrit entre le XIIème et le XIVème siècle, sur le passé de l’Islande, mais également de la Scandinavie. Ces récits étaient considérés, à cette époque, comme une grande source de renseignement sur leur histoire au Moyen-Âge et étaient jugés comme étant des récits véridiques. Les différents historiens danois et suédois de l'époque les considéraient comme étant des sources fiables sur le passé de leurs royaumes respectifs.

Dans ses études préparatoires, Olaus Rudbeck fut frappé par des ressemblances étonnantes entre les noms de personnes, de lieux et de peuples contenus dans cette saga et ceux qu'il avait auparavant lu dans des textes antiques pendant sa jeunesse. Ce fut le point de départ de son raisonnement. Dans sa correspondance avec son ami Magnus Gabriel De la Gardie, Olaus Rudbeck exprima tout son enthousiasme, parce qu'il estimait qu'il avait découvert la véritable identité de la Suède, qui était en réalité l'Atlantide des mythes antiques. Il mentionnait qu'il avait trouvé chez les auteurs grecs et latins «de telles antiquités pour notre patrie que c'en était proprement stupéfiant» ${ }^{17}$. Quoi que puissent dire les plus grands érudits européens, le berceau de la culture s'était jadis trouvé en Suède, et non à Rome ou en Grèce. La Suède était le foyer originel du

\footnotetext{
14 DAHLGREN, Stellan, Karl XI in FLORÉN, Anders ; DAHLGREN, Stellan \& LINDEGREN, Jan (red.), op. cit.

${ }_{15}$ FRÄNGSMYR, Tore, A la recherche des Lumières: une perspective suédoise, Bordeaux, Presses universitaires de Bordeaux, 1999.

${ }^{16}$ ERIKSSON, Gunnar, Rudbeck 1630-1702: liv, lärdom, dröm i barockens Sverige, Stockholm, Atlantis, 2002.

${ }^{17}$ ANNERSTED, Claes : Olof Rudbeck den äldre : kort lefnadstekning; Stockholm, Nordstedt, 1905, p109
} 
savoir et de la sagesse humaine, selon Olaus Rudbeck, et cela démontrait que le roi de Suède faisait partie des plus prestigieux souverains.

Le roi Charles XI vit dans cet ouvrage une très belle occasion pour redorer son image, tant auprès du peuple qu'auprès des grandes cours européennes. Olaus Rudbeck rédigea son ouvrage uniquement en suédois, mais avant la publication il fut convaincu d'y ajouter la traduction en latin, pour le rendre accessible aussi bien aux Suédois qu'au reste de l'Europe. En 1675, Magnus Gabriel de la Gardie, son protecteur, lui obtint une aide financière pour la publication d'Atlantica, mais Olaus Rudbeck étudiait une littérature très riche et dense, qui soulevait de nombreux problèmes. Il fit finalement publié le premier volume d'Atlantica au printemps 1679, quelques mois avant la signature du traité de Fontainebleau, qui mettait fin à la guerre de Scanie entre le Danemark et la Suède.

Ce premier volume était conçu comme un tout, sans qu'une suite soit prévue, mais la littérature tellement riche et les perspectives prestigieuses lui firent changer d'avis pendant la rédaction du premier volume, ainsi que l'accueil favorable réservé à Atlantica dans les milieux savants étrangers. Cela stimula l'ambition d'Olaus Rudbeck. En 1689, il publia le deuxième volume, puis le troisième en 1698 mais il n'eut pas le temps de finir la rédaction du quatrième volume et mourut avant, en octobre 1702. Ce volume inachevé fut publié immédiatement après sa mort. Mais l'essentiel de l'argumentation d'Olaus Rudbeck se trouve dans le premier volume d'Atlantica. Sa méthode historique rassembla les preuves prouvant l'âge fabuleux du royaume de Suède et sa véritable identité cachée: l'Atlantide ensevelie et les autres terres de l'âge d'or des mythes antiques.

Il était important de donner une image du roi de grand souverain guerrier. La guerre de Scanie épuisait la population suédoise, qui subissait directement les combats. Les troupes danoises et suédoises s'affrontaient dans le sud de la Suède, qui était l'enjeu principal de cette guerre. Charles XI avait déjà obtenu une grande victoire militaire sur les Pays-Bas, et après sa victoire sur les troupes danoises lors de la bataille de Lund en 1679, il obtint un grand prestige militaire et une popularité importante auprès de la population suédoise. Il se présentait comme le vrai défenseur de la Suède, alors que la population était en colère contre le comportement de la noblesse pendant la guerre de Scanie, qu'elle qualifiait de lâche.

Il était important pour le roi de pouvoir s’identifier avec les héros mythiques pour renforcer sa position sur le trône de Suède, obtenir les pleins pouvoirs par rapport au conseil du royaume et continuer sa propagande contre l'ennemi danois. Alors, pour redonner toute sa splendeur historique à la Suède et à son roi, Olaus Rudbeck reprit les 
grands mythes antiques classiques un à un pour leur donner un aspect nordique achevé. Il récupéra tous les héros et mythes qu'il pouvait, afin de leur donner une touche suédoise, voir si possible de les suédiser complètement, et ainsi donner au royaume une légitimité et une supériorité historiques incontestables, en particulier face au Danemark.

À partir de ses recherches, il avait établi que toutes les mythologies prenaient leurs origines dans la mythologie nordique, qui se déroulait généralement en Suède, mais surtout pas au Danemark. Il expliqua aussi scientifiquement que certains épisodes des grands récits antiques ne pouvaient s'être déroulés qu'en Suède, même si la Suède n'était jamais mentionnée précisément, et que les dieux et les grands héros antiques étaient souvent d'origine suédoise.

\section{La Suède des Hyperboréens}

Pour commencer, Olaus Rudbeck se basa sur le mythe des Hyperboréens, raconté par Platon, dans son ouvrage le Timée et dans un fragment du Critias. Connu par les récits de Platon, les Hyperboréens étaient présentés comme un peuple, qui habitait aux confins septentrionaux du monde civilisé, décrits comme des voyageurs venus du Nord. Leur terre, connue sous le nom d'Hyperborée, était considérée comme parfaite, où le soleil brillait constamment. Au sens étymologique, cela caractérisait ceux qui vivent “au-delà de Borée, le vent du nord". Ils étaient considérés comme une peuplade instruite, qui n'avait peur de rien, possédant la plus grande sagesse, dans une société considérée comme idéale et parfaite. Les Hyperboréens étaient un symbole très fort, notamment grâce à Platon, qui, dans ses récits, faisait leur éloge.

Nombreux sont les savants suédois, qui se sont intéressés à l'historiographie de la Suède, en se basant sur le mythe des Hyperboréens, les situant, à chaque fois, dans le nord de l'Europe, et plus particulièrement en Suède, en se basant sur les données géographiques, astronomiques et géologiques énoncées par Platon. En effet, Platon caractérisait ce fameux pays, l'Hyperborée, comme l'endroit où le soleil brillait tout le temps pendant une partie de l'année et, pendant l'autre, la lune le remplaçait. Les Hyperboréens vivaient au pays du soleil de minuit, un phénomène typiquement nordique. Ce peuple était décrit comme étant heureux, sans maladie, n'ayant que des vertus, et sans aucun vice. La légende des Hyperboréens et un nombre considérable de témoignages avaient entraîné Olaus Rudbeck sur le fait que l'ancienne Suède avait été considérée comme le paradis sur terre, à partir duquel les dieux, les arts et les sciences étaient apparues, avant de se répandre dans le monde. 
En plus des descriptions géographiques, Rudbeck se servit de la position des étoiles, décrites par Platon, pour situer le pays des Hyperboréen. À partir de cela, il put estimer que l'Hyperborée, pays situé au nord de la Grèce Antique, ne pouvait se situer qu'en Scandinavie, dans le nord de la Scandinavie, mais surtout pas au Danemark, notamment à cause de la latitude élevée. Il compara avec ce qu'il pouvait observer dans le ciel suédois.

Nous pouvons observer sur les cartes, qu'Olaus Rudbeck ajouta à son ouvrage, qu'il intégrait la Norvège comme faisant naturellement partie de la Suède, sous la même appellation Hyperborée. Mais le Danemark n'est pas nommé, dans une volonté d'essayer de l'intégrer aux pays celtes, rendant ainsi impossible pour le Danemark d'être le pays des Goths. Il mettait ainsi en avant les ambitions politiques suédoises de conquérir la Norvège, alors une partie du royaume de Danemark, et se servait de ses théories pour légitimer les prétentions suédoises sur cette région, en mettant en évidence que la Norvège a été une partie naturelle et historique du royaume de Suède.

Atlantica était une œuvre de propagande politique, dont le but principal était de prouver que la Suède avait toujours été ce grand royaume très puissant, auquel le roi Gustave II Adolphe avait rendu sa splendeur passée. Les intentions politiques d'Olaus Rudbeck étaient visibles, malgré sa réelle envie de découvrir un vrai passé nordique universel en Suède. Son œuvre est très complexe, tant dans sa méthode que dans ses sources. Il faut mettre en évidence les sources sur lesquelles il a basé sa réflexion, sans oublier de mettre en rapport le but politique d'Olaus Rudbeck pour comprendre la portée de cet ouvrage. Son but principal était de légitimer la monarchie suédoise aux yeux du peuple, mais également au niveau des grandes cours européennes.

\section{La Suède des mythes antiques classiques}

Olaus Rudbeck remarqua les nombreuses similitudes entre certains faits rapportés dans les mythes antiques et la réalité suédoise. À partir de ses premières estimations, il comprit que la littérature classique antique devait très certainement receler de nombreux témoignages sur les Hyperboréens, leur pays, leur culture, leurs mœurs, et que cela pouvait donner des indications essentielles pour redécouvrir le véritable passé de la Suède.

Il a pu trouver dans des récits sur les grands héros antiques des détails, qui s'apparentaient parfaitement ou presque à la Suède. Il profita de ces petits détails pour pouvoir s'approprier ces célèbres héros et les transposer à son royaume. Il reprit tous les grands mythes et grands héros qui existaient et qui étaient connus et les transposa 
aux grands mythes suédois, dans le but de leur rendre leur véritable identité suédoise, ou bien il transposait quelques-unes des grandes aventures héroïques, pour faire en sorte que l'action se déroule en Suède. Pour faire cela, il se servit plus spécifiquement des descriptions géographiques et topographiques des paysages, de la position des étoiles et détermina qu'il ne pouvait s'agir que de la Suède. D’après les théories de Rudbeck, toutes les grandes mythologies d'Europe étaient liées à l'histoire de la Suède.

Il fut également capable d'établir que Ulysse était passé par la Suède lors de son voyage. Il avait attentivement étudié les grands textes classiques, en particulier ceux d'Homère, qui lui apportèrent une grande satisfaction intellectuelle dans ses recherches. Par exemple, Ulysse était connu pour sa longue errance dans l'Odyssée, vivant de grandes aventures très périlleuses, mais très prestigieuses. Olaus Rudbeck étudia toutes les descriptions du voyage d'Ulysse, jusqu'au moment où il en trouva une, dont les paysages correspondaient exactement à la Suède d'après ses estimations.

Ulysse aborda une île avec des forêts importantes et de nombreux lacs, l'île de Calypso, où il resta prisonnier pendant sept années. Calypso était une nymphe, fille d'Atlas, qui dirigeait l'île d'Ogygie. Située très loin dans la mer, au bout du monde selon Homère, l'île présentait une végétation très riche et variée, ainsi qu'une grande variété d'oiseaux. La perfection de cette île de délice était telle que «dès l'abord en ces lieux, même un dieu aurait eu les yeux charmés et l'âme ravie». Dans le Chant 1 de l'Odyssée, Calypso est nommée comme étant la «fille d'Atlas, qui connaît les abîmes de la mer entière et veille sur les hautes colonnes écartant l'un de l'autre le ciel et la terre». Pour Olaus Rudbeck, l'île de Calypso correspondait également à l'île d'Atlas.

Après cela, il estima que d'autres héros de la mythologie grecque pouvaient également avoir des liens avec la Suède, comme le récit des douze travaux d'Hercules. Il estima que le onzième travaux d'Hercules s'était déroulé en Suède, celui des pommes du jardin des Hespérides. Les Hespérides étaient les filles d'Atlas et vivaient dans un jardin aux confins du monde. Ces pommes avaient été données par Gaïa à Héra comme cadeau de noces. Conservées dans le jardin des Hespérides, elles étaient le symbole de la sagesse et de la puissance, et elles étaient très convoitées. Seul Atlas pouvait s'en saisir. Ce jardin était considéré comme un jardin d’immortalité, réservé aux dieux. Olaus Rudbeck estimait cela comme un grand honneur et une véritable marque de prestige le fait qu'un de ses exploits les plus mythiques de ce grand héros mythologique se soit, d'après ses estimations, déroulé en Suède. Les descriptions de ce jardin correspondaient exactement aux paysages suédois, tant dans la hauteur des montagnes que dans la disposition des fleuves. 
En réalisant ses recherches, il remarqua aussi que l'étymologie du nom grec Héraklès et son équivalent romain Hercules avaient des origines suédoises. La première signification, à partir du nom grec, démontrait que Hera provenait du suédois här, c'est à dire armée, klès de klädd, c'est à dire vêtement. La signification finale donnait le vêtement de l'armée, c'est à dire l'armure, symbole de protection et d'invincibilité. La seconde signification se basait sur le nom romain Hercule et signifiait Her pour här, en suédois, et kull, signifiant tête. Cela signifiait simplement le chef d'une armée. D'un point de vue strictement linguistique, Olaus Rudbeck estimait que Héraklès et Hercules, qui ont la même origine, étaient obligatoirement d'origine suédoise.

Il employa la même méthode avec les noms des dieux grecs notamment. Par exemple, le nom de la déesse Vénus venait du mot suédois vän, signifiant joli, gracieux. Sur le même modèle, Chimène provenait du mot suédois skinnmära, signifiant rosse, haridelle et la Thèbes égyptienne était en réalité la ville suédoise de Täby. Sur le même modèle, le mot grec Elyséen venait d'une déformation du mot suédois glysisvall, de glysa - briller. Le pays des Cimmériens d'Homère venait du mot suédois Kämparnäs, signifiant le cap des guerriers. Atlantica regorge d'explications de ce même genre, prouvant que l'étymologie des mots est suédoise ${ }^{18}$.

Ses recherches le ramenaient à chaque fois à l'île d'Atlas, le menant directement au célèbre mythe de l'Atlantide. Du grec ancien signifiant l'île d'Atlas, l'Atlantide est une île qui aurait été engloutie dans la pré-Antiquité. l'Atlantide est mentionnée pour la première fois par Platon dans le Timée, puis dans le Critias. Platon précisait dans ses dialogues «qu'il ne s'agit pas d'une fiction, mais d'une histoire véritable». Le mot île, en grec ancien nesos, pouvait également signifier presqu'île, ce qui arrangeait bien Rudbeck. À ses yeux, la presqu'île de la Scandinavie remplissait toutes les conditions définies par Platon, mais cela ne comprenait pas le Danemark et il ne planait aucun doute sur le fait que la Suède était bien l'Atlantide.

Selon les récits rapportés par Platon, l'Atlantide était une île fortement peuplée et militarisée, située face aux Colonnes d'Hercule, qui sombra dans la mer après le violent combat perdu par les Atlantes contre les Athéniens. Platon décrit l'Atlantide et son organisation sociale de manière concrète. Le dieu des mers Poséidon, qui avait reçu l'île en partage, y vivait avec son épouse et ses dix fils dans sa forteresse entourée de trois bras de mer.

${ }^{18}$ ERICSSON, Gunnar, The Atlantic Vision: Olaus Rudbeck and Baroque Science, Canton, MA, Science history publications, 1994. 
Tous les indices platonniens correspondaient à la géographie et la topographie de la Suède. Par exemple, Platon avait expliqué que l'île de l'Atlantide se situait après le passage des colonnes d'Hercule, symbole de la frontière entre le monde civilisé et un monde inconnu et dangereux. Cet élément était le plus distinctif de ces descriptions. Olaus Rudbeck détermina que les colonnes d'Hercule n'étaient pas le détroit de Gibraltar, comme tout le monde le pensait, mais en réalité le détroit de l'Öresund. Les caractéristiques et les descriptions pouvaient s'adapter parfaitement au détroit de l'Öresund, mieux qu'au détroit de Gibraltar. Bien que jamais situées précisément dans les différents témoignages, pour lui tous les détails correspondaient en réalité aux paysages suédois. De plus, avant que le terme géographique de Colonnes d'Hercule ne s'imposent au VIème siècle, les Grecs employaient celui de Colonnes d'Atlas, qui avait plus une fonction cosmologique. Et Atlantide signifiant île d'Atlas, Olaus Rudbeck avait déjà démontré que l'île d'Atlas correspondait au pays des Hyperboréens, donc à la Suède.

\section{Atlantica, d'abord une ouvre politique}

Grâce à Platon et sa renommée, Rudbeck put installer un rayonnement culturel important dans son œuvre Atlantica, donnant ainsi à l'histoire suédoise un statut prestigieux et solide d'un point de vue intellectuel et politique. D’après le récit de Platon, l'Atlantide était une grande puissance militaire, politique et sociale. C'était une île plus avancée que le continent européen, dans tous les domaines. Le système politique était un exemple de perfection, son armée était la plus puissante que le monde ait jamais vu. Les habitants vivaient heureux, en harmonie. Le système social était également un exemple de perfection, ne laissant personne sur le bord de la route. L’Atlantide était présentée par Platon comme la terre idéale, où tout était parfait. Il s'agissait de la propagande parfaite pour mettre son royaume en valeur et lui donner une légitimité inattaquable.

Il récupéra tous les héros et mythes, qu'il pouvait, afin de leur donner une touche suédoise, voir si possible de les suédiser complètement, et ainsi donner au royaume une légitimité et une supériorité historiques incontestables, en particulier face au Danemark. À partir de ses recherches, il avait établi que toutes les mythologies prenaient leurs origines dans la mythologie nordique, qui se déroulait généralement en Suède, et surtout pas au Danemark. Il expliqua aussi scientifiquement que certains épisodes des grands récits antiques ne pouvaient s'être déroulés qu'en Suède, même si 
la Suède n'était jamais mentionnée précisément, et que les dieux et les grands héros antiques étaient souvent d'origine suédoise.

Aucune difficulté ne résistait à Olaus Rudbeck avec son épuisable capacité inventive. Lorsque Platon parlait de vin et d'éléphants dans l'Atlantide, Rudbeck avait une réponse toute prête : par vin, il fallait entendre bière ou hydromel, et les éléphants étaient des loups. L'engloutissement de l'Atlantide dans la mer ne faisait que désigner métaphoriquement l'état désolé du pays après les premiers grands exodes. L'île était toujours présente dans le Nord, chacun pouvait venir la contempler, surtout à présent qu'elle était redevenue une grande puissance militaire et politique.

La Suède n'était jamais nommée explicitement, mais ce n'était pas un problème pour Rudbeck. Il savait qu'il s'agissait bien de son pays. Les descriptions cadraient parfaitement avec la topographie du pays. Il estimait qu'il était écrit quelque chose, mais que cela signifiait autre chose. Les mots de Sten Lindroth, professeur émérite d'histoire des idées à l'université d’Uppsala, résument bien la conception d'Olaus Rudbeck de son travail:

\begin{abstract}
Pour Rudbeck, possédé par son idée, aucun texte n’offrait de telles résistances qu'il ne sût le tourner à son profit. La discipline méthodologique qu'il s'était imposé comme anatomiste n'était plus qu'un souvenir lorsqu'il faisait revivre l'Atlantide de jadis. Il n'y a pas lieu pour autant d'y voir une énigme psychologique. En tant qu'historien gotique, Rudbeck avait emprunté d'autres manières de procéder et habitudes mentales - simplement, tout ce qu'il touchait prenait aussitôt des proportions monumentales ${ }^{19}$.
\end{abstract}

Olaus Rudbeck était un homme très hétéroclite et cela se reflète dans son œuvre Atlantica, où il allia à la fois passé, avec les sources historiques, et futur, avec ses méthodes scientifiques visionnaires, pour obtenir le résultat qu'il voulait. Olaus Rudbeck ne s'arrêta pas uniquement aux textes classiques de l'Antiquité, et s'attaqua à l'ouvrage le plus important et le plus connu du moment : la Bible. Il en étudia de près tous les détails des descriptions et réussit à établir, de la même manière qu'il l'avait fait avec l'Atlantide, que la Suède était également le jardin d'Éden. Il argumenta l'origine suédoise des noms d'Adam, originaire du mot suédois damm, signifiant poussière, et Ève, Eva en suédois, venant de l'expression Eh vad, signifiant à quoi, qui proviendrait du hurlement que poussa Adam lorsqu'une de ses côtes lui fut prise pour créer la femme. Selon ses réflexions, le jardin d'Éden se trouvait en Suède, qui était aussi la

19 LINDROTH, Sten, Svensk lärdomhistoria: Stormaktstiden, vol. 2, Stockholm, P.A. Norstedt, 1975, p. 327. 
représentation du paradis, symbolisé par les journées sans fin de l'été suédois, et de l'enfer, par les nuits sans fin de l'hiver suédois.

\section{Conclusion}

Olaus Rudbeck avait prévu d'écrire son histoire de la Suède en neuf volumes. Mais il mourut en octobre 1702, alors qu'il venait juste de commencer la rédaction du quatrième volume d'Atlantica. Ce volume inachevé fut publié immédiatement après sa mort. Cette nouvelle histoire nationale de la Suède fut une véritable révolution lors de la parution du premier volume. Elle permettait à ce royaume à la position très septentrionale, de se mettre en lumière auprès des grandes cours européennes et dans les cercles de savants et d'intellectuels. Mais, lors de la publication des trois volumes suivants, les critiques devinrent plus vives à l'encontre du contenu de l'ouvrage.

Malgré le fait que depuis la publication du second volume d'Atlantica les savants et les intellectuels des grandes cours d'Europe ne prenaient plus du tout au sérieux Olaus Rudbeck et son ouvrage, le nouveau roi de Suède était très attaché à l'image qu'il avait créé de la Suède. Cette image renforcée du royaume de Suède et de ses souverains à partir d'une histoire universellement reconnue apporta beaucoup de prestige au nouveau roi Charles XII. Sten Lindroth, qualifiait Atlantica dans ces termes:

\footnotetext{
Atlantica se dresse comme le monument, à l'ère de grande puissance, de la recherche patriotique égarée, monument inquiétant et presque effrayant par le mélange qu'il offre de génie et de folie. Pour ce qui est de la grandeur de la vision et de l'énorme énergie déployée pour donner corps à celle-ci, cet ouvrage est sans équivalent dans l'histoire du savoir en Suède ${ }^{20}$.
}

En 1697, Charles XI mourut et son fils Charles XII fut couronné. Il commença à poser les bases de la grande guerre du Nord, qui ébranla toute l'Europe du Nord entre 1700 et 1721 . Ce roi fut connu pour ses exploits militaires, qui furent rapportés dans l'Histoire de Charles XII de Voltaire, publiée en 1731. Son règne fut marqué par la grande guerre du Nord, lors de laquelle Charles XII de Suède déclara la guerre à tous ses voisins du Nord. Cette guerre marqua la fin de la période de grandeur de la Suède, devenant alors un royaume de second plan en Europe.

Atlantica a probablement aidé le roi Charles XII à développer ses prétentions personnelles et militaires, lui démontrant que la puissance d'un royaume tenait dans

${ }^{20}$ LINDROTH, Sten, op. cit., p. 330. 
son histoire et ses conquêtes militaires. Grâce à Atlantica, il pouvait se définir comme descendant d'une grande lignée historique, originaire du pays, qui avait inventé l'écriture et la science. Cette conscience de sa supériorité par rapport au reste du monde se retrouva pendant tout son règne, qui ne fut marqué que par des guerres de conquêtes, pour accroître le royaume de Suède et son prestige. Le roi Charles XII mourut au combat en 1718 en Norvège et le royaume de Suède perdit son statut de grande puissance d'Europe du Nord, ainsi que ses colonies dans l'espace Baltique et dans le nord de l'Allemagne. Ce fut la fin de la période de grandeur de la Suède, qui ne retrouva plus jamais cette grandeur et ce prestige atteints. 


\section{* L'auteur}

Muriel Marchal, doctorante en études nordiques, travaille sur les relations entre le Danemark et la Suède entre 1563 et 1770 . Elle est spécialiste en histoire moderne politique, diplomatique et des idées en Scandinavie. Sa thèse porte sur la construction des identités nationales scandinaves, à travers la propagande nationale et la diplomatie scandinave, allant de l'hostilité à la réconciliation neutre entre les deux royaumes scandinaves.

URL: < http://www.studistorici.com/progett/autori/\#Marchal >

\section{Per citare questo articolo:}

MARCHAL, Muriel, «Légitimer le pouvoir royal suédois à travers une nouvelle construction de l'histoire nationale de Suède», Diacronie. Studi di Storia Contemporanea : Periferie. Cultura, economia, politica, 29/3/2014, URL: < http://www.studistorici.com/2014/3/29/marchal_numero_17/ >

Diacronie Studi di Storia Contemporanea www.diacronie.it

Risorsa digitale indipendente a carattere storiografico. Uscita trimestrale. redazione.diacronie@hotmail.it

Comitato di redazione: Jacopo Bassi - Luca Bufarale - Elisa Grandi - Deborah Paci - Fausto Pietrancosta - Matteo Tomasoni - Luca Zuccolo

Diritti: gli articoli di Diacronie. Studi di Storia Contemporanea sono pubblicati sotto licenza Creative Commons 2.5. Possono essere riprodotti a patto di non modificarne i contenuti e di non usarli per fini commerciali. La citazione di estratti è comunque sempre autorizzata, nei limiti previsti dalla legge. 